\section{D) Check for updates}

Cite this: Analyst, 2020, 145, 6586

\title{
Cheating on forensic hair testing? Detection of potential biomarkers for cosmetically altered hair samples using untargeted hair metabolomics $\uparrow$
}

\author{
Lisa Eisenbeiss, ${ }^{a}$ Tina M. Binz, (DD ${ }^{b}$ Markus R. Baumgartner, (D) ${ }^{b}$ Thomas Kraemer (D) ${ }^{a}$ \\ and Andrea E. Steuer (D)*a
}

\begin{abstract}
Hair analysis has become an integral part in forensic toxicological laboratories for e.g. assessment of drug or alcohol abstinence. However, hair samples can be manipulated by cosmetic treatments, altering drug concentrations which eventually leads to false negative hair test results. In particular oxidative bleaching of hair samples under alkaline conditions significantly affects incorporated drug concentrations. To date, current techniques to detect cosmetic hair adulterations bear limitations such as the implementation of cut-off values or the requirement of specialized instrumentations. As a new approach, untargeted hair metabolomics analysis was applied to detect altered, endogenous biomolecules that could be used as biomarkers for oxidative cosmetic hair treatments. For this, genuine hair samples were treated in vitro with 9\% hydrogen peroxide $\left(\mathrm{H}_{2} \mathrm{O}_{2}\right)$ for 30 minutes. Untreated and treated hair samples were analyzed using liquid-chromatography high-resolution time-of-flight mass spectrometry. In total, 69 metabolites could be identified as significantly altered after hair bleaching. The majority of metabolites decreased after bleaching, yet totally degraded metabolites were most promising as suitable biomarkers. The formation of biomarker ratios of metabolites decreasing and increasing in concentrations improved the discrimination of untreated and treated hair samples. With the results of this study, the high variety of identified biomarkers now offers the possibility to include single biomarkers or biomarker selections into routine screening methods for improved data interpretation of hair test results.
\end{abstract}

\begin{abstract}
Received 24th June 2020, Accepted 6th August 2020 DOI: $10.1039 / \mathrm{d} 0 \mathrm{an} 01265 \mathrm{c}$ rsc.li/analyst
\end{abstract}

\section{Introduction}

Over the past years, hair analysis has become increasingly important. Because of its non-invasive collection, easy-storage and in particular its long-term detection window, hair is the matrix of choice in forensic toxicology to assess retrospective consumption behaviors. It is therefore routinely applied for the assessment of drugs of abuse (DOA) or alcohol abstinence, workplace drug testing and in childhood custody cases. ${ }^{1}$ Yet, drug or alcohol concentrations can significantly be affected by the application of cosmetic hair treatments. These treatments include "detox-shampoos", tinting, semi-permanent/permanent hair dyeing or hair straightening, ${ }^{2-7}$ however the most effective being oxidative hair treatments with hydrogen per-

\footnotetext{
${ }^{a}$ Department of Forensic Pharmacology and Toxicology, Zurich Institute of Forensic Medicine, University of Zurich, Zurich, Switzerland.

E-mail: andrea.steuer@irm.uzh.ch; Fax: +41446356852; Tel: +41 446355679

${ }^{b}$ Center for Forensic Hair Analytics, Zurich Institute of Forensic Medicine, University of Zurich, Zurich, Switzerland

$\dagger$ Electronic supplementary information (ESI) available. See DOI: 10.1039/ d0an $01265 \mathrm{c}$
}

oxide $\left(\mathrm{H}_{2} \mathrm{O}_{2}\right)$ under alkaline conditions which are used for permanent hair dyeing and hair bleaching. ${ }^{8,9}$ Especially the latter can decrease DOA or alcohol marker concentrations significantly and might potentially result in false negative hair testing results. ${ }^{10}$ This makes them popular for intended hair manipulation to avoid positive tests.

A major challenge in routine hair analysis is the detection of such adulteration attempts. So far, visual inspections of hair samples and colored extracts can give rise to suspected cosmetic hair treatments. Objective markers would however improve confidence in adulteration confirmation, particularly in court. For that reason, more complex techniques such as fluorescence microscopy ${ }^{11}$ or infrared spectroscopy ${ }^{12}$ have been developed over time to detect cosmetically treated hair samples. Yet, the detection of adulteration attempts with routinely available procedures like liquid chromatography tandem mass spectrometry (LC-MS/MS) would be ideal. In a first study, $1 H$-pyrrole-2,3,5-tricarboxylic acid (PTCA) was analyzed by LC-MS/MS and described as a marker for oxidative hair treatments. ${ }^{13}$ However, the implementation of cut-off values for PTCA is indispensable. Recently, $1 H$-pyrrole-2,3,4,5-tetracarboxylic acid (PTeCA) was identified to be exclusively formed 
through oxidative hair treatment which would in theory facilitate the detection of bleached hair samples as cut-off values do not need to be determined. ${ }^{14}$ At the moment, the current lack of commercially available reference standards and its melanin dependency still hinders its routine applicability. Hence, the need for further reliable markers for cosmetic hair treatments is more than relevant to date and new concepts for biomarker search are required.

The detection of direct oxidation products of DOA could be thought of as has been proposed for 2-nitro-6-monoacetylmorphine and 2-nitro-morphine as markers for $\mathrm{KNO}_{2}$ adulterated urine samples. ${ }^{15,16}$ Yet, this would require evaluation for every drug once again. A drug-independent, endogenous marker or a set thereof would therefore be better suited. As oxidative hair treatments can significantly affect DOA incorporated into the hair matrix, an impact on endogenous compounds in the same or similar way is likely.

Metabolomic analyses aim to extensively study small endogenous metabolites of an organism under different conditions (e.g. healthy vs. disease) to describe physiological processes and identify biomarkers. ${ }^{17}$ An untargeted metabolomics approach in theory analyses all metabolites without prior knowledge and serves as a hypothesis-generating tool. In recent studies by Joo et al. and Grosvenor et al., the influence of hair bleaching on amino acids and lipids and proteins was investigated, respectively. ${ }^{18,19}$ The authors could show that oxidative hair treatment has a significant impact on the studied compounds. However, these studies were conducted for a small selection of endogenous compounds and aimed to analyze the impact of hair bleaching on hair damage. Hence, applying an untargeted metabolomics approach should broaden the number of metabolites that potentially reveal differences of untreated and oxidatively treated hair samples and detect endogenous biomarkers that indicate manipulation attempts within routine hair analysis.

Within this proof-of-concept study, we aimed to identify a variety of possible biomarkers or biomarker combinations for oxidative hair treatments which could then potentially be applied within standard routine screening methods. For this, we applied an untargeted hair metabolomics approach to find altered and stable (for normalization purposes) endogenous metabolites between cosmetically untreated and bleached hair samples.

\section{Materials and methods}

\subsection{Chemicals and reagents}

1-Methylhistidine, $\varepsilon$-acetyllysine, adenine, adenosine, arginine, aspartic acid, azelaic acid, butyrylcarnitine (C4), chenodeoxycholic acid, cholic acid, choline, citrulline, cortisol, cortisone, creatinine, cysteic acid, DL-indole-3-lactic acid, decanoylcarnitine (C10), deoxycholic acid, glutamic acid, glutaric acid, glycocholic acid, hexanoylcarnitine (C6), hippuric acid, histidine, hypoxanthine, inosine, isoleucine, L-carnitine (C0), L-cysteine$S$-sulfate, leucine, lysine, methionine, methylmalonic acid, mevalonolactone, $\mathrm{N}$-acetylneuraminic acid, $\mathrm{N}, \mathrm{N}$-dimethylglycine, nicotinamide, nicotinic acid, octanoylcarnitine (C8), ornithine, $p$-aminobenzoic acid, phenylalanine, proline, propionylcarnitine (C3), pyroglutamic acid, raffinose, riboflavin, serine, suberic acid, taurine, taurocholic acid, theobromine, theophylline, threonine, trimethyllysine, tryptophan, tyrosine, uracil, uric acid, urocanic acid and valine, were purchased as analytical standards from Sigma-Aldrich (Buchs, Switzerland). The analytical reference standard of PTCA was sourced from ChemPur (Karlsruhe, Germany) and PTeCA was synthesized and delivered by ReseaChem Life Science (Burgdorf, Switzerland). All heavy-labeled and deuterated internal standards (IS) adenosine ribose- $\mathrm{D}_{1}$, arginine $-{ }^{13} \mathrm{C}_{6}$, caffeine 3-methyl $-{ }^{13} \mathrm{C}$, carnitine trimethyl- $\mathrm{D}_{9}$, deoxycholic acid- $\mathrm{D}_{4}$, D-fructose ${ }^{13} \mathrm{C}$, hippuric acid ${ }^{15} \mathrm{~N}$, leucine- $\mathrm{D}_{10}$, lysine- $\mathrm{D}_{4}$, phenylalanine- $\mathrm{D}_{1}$, proline ${ }^{15} \mathrm{~N}$ and tryptophan- $\mathrm{D}_{5}$ were purchased from Cambridge Isotope Laboratories, Inc. (Andover, MA, USA) and delivered by ReseaChem Life Science (Burgdorf, Switzerland) or Sigma-Aldrich (Buchs, Switzerland). Water, acetonitrile (ACN), dichloromethane (DCM) and methanol $(\mathrm{MeOH})$ of HPLC grade were obtained from Fluka (Buchs, Switzerland). All other chemicals used were from Merck (Zug, Switzerland) and of the highest grade available.

Bleaching reagents (Venice Oxydant \& active lotion containing $9 \% \quad \mathrm{H}_{2} \mathrm{O}_{2}$; Venice bleaching oxidation powder) were obtained from DOBI (Suhr, Switzerland) and commercial shampoo (I am, Intense Moisture, Buchs, Switzerland) from a local store.

\subsection{Hair samples}

Hair samples without cosmetic hair treatment $(n=21$, analysis of the proximal $3 \mathrm{~cm}$ segment only) were used within this study (14 male and 7 female hair samples aged between 19 and 54). Hair sample colors ranged from black $(n=4)$, over dark brown to medium brown $(n=7)$, light brown to dark blond ( $n=5)$, to medium blond to light blond $(n=5)$. Hair samples were obtained from volunteers who provided written informed consent and from the Center for Forensic Hair Analytics (Zurich, Switzerland) from routine case work after anonymization. Samples taken proactively from volunteers did not require an additional ethical approval according to the Swiss law (Humanforschungsgesetz) if the research is not aiming to investigate diseases or functions of the human body as is the case in the current study. Reuse of authentic samples from human case work was in full conformance with Swiss laws according to the statement of Cantonal Ethics Board of the Canton of Zurich (BASEC-no. Req-2017-00946). Samples were collected close to the scalp and stored in aluminum foil at room temperature.

\subsection{General hair adulteration procedure}

Cosmetically untreated hair samples were split into two portions of which one was left untreated and one was bleached in vitro according to the manufacturer's instructions with a mixture of $2 \mathrm{~g}$ bleaching oxidase (containing $9 \% \mathrm{H}_{2} \mathrm{O}_{2}$ ) and $1 \mathrm{~g}$ bleaching oxidation powder during incubation for $30 \mathrm{~min}$. 
After initial rinsing of bleached hair samples to reach a neutral $\mathrm{pH}$ of the washing solution, untreated and bleached hair samples were both rinsed under running water and subsequently with $500 \mu \mathrm{L}$ shampoo solution (1 mL shampoo, $9 \mathrm{~mL} \mathrm{H}_{2} \mathrm{O}$ ). Finally, hair samples were dried at room temperature overnight and processed as described in the following section 2.4.

\subsection{Sample preparation}

All hair samples were prepared as described in detail elsewhere $^{20}$ with minor changes. Hair samples were successively washed with DCM, acetone, $\mathrm{H}_{2} \mathrm{O}$ and acetone and dried overnight. $31.5 \pm 0.5 \mathrm{mg}$ of hair was weighed into a $2 \mathrm{~mL}$ Eppendorf tube containing one tungsten carbide ball and pulverized (10 $\mathrm{min}$ at $30 \mathrm{~Hz}$ ) using a bench-top mill (Mixer Mill MM 400, Retsch, Haan, Germany). Hair samples were extracted with $1 \mathrm{~mL} \mathrm{ACN} / \mathrm{H}_{2} \mathrm{O}(2: 8, \mathrm{v} / \mathrm{v}$ ) and $20 \mu \mathrm{L}$ IS solution (adenosine ribose- $\mathrm{D}_{1}(0.075 \mathrm{mM})$, arginine $-{ }^{13} \mathrm{C}_{6}(1.5 \mathrm{mM})$, caffeine 3-methyl- ${ }^{13} \mathrm{C}$ (1 mM), carnitine trimethyl- $\mathrm{D}_{9}(0.5 \mathrm{mM})$, deoxycholic acid- $\mathrm{D}_{4}(0.009 \mathrm{mM})$, D-fructose ${ }^{13} \mathrm{C}(0.6 \mathrm{mM})$, hippuric acid ${ }^{15} \mathrm{~N}(2.5 \mathrm{mM})$, leucine- $\mathrm{D}_{10}(1.5 \mathrm{mM})$, lysine- $\mathrm{D}_{4}(3.5 \mathrm{mM})$, phenylalanine- $\mathrm{D}_{1}(1.5 \mathrm{mM})$, proline ${ }^{15} \mathrm{~N}(3.5 \mathrm{mM})$ and tryptophan- $\mathrm{D}_{5}(1.25 \mathrm{mM})$ ) during $16 \mathrm{~h}$ of incubation in an ultrasonic bath. Afterwards, samples were centrifuged ( $5 \mathrm{~min}, 9^{\prime} 000 \mathrm{rpm}$ ) and the supernatant $(900 \mu \mathrm{L})$ was evaporated to dryness under nitrogen at $35^{\circ} \mathrm{C}$. After reconstitution of the dried extract with $250 \mu \mathrm{L} \mathrm{ACN} / \mathrm{H}_{2} \mathrm{O}(2: 8, \mathrm{v} / \mathrm{v})$, samples were further purified by combined centrifugation and filtration (5 min, $9000 \mathrm{rpm}$, VWR centrifugal filter, $0.45 \mu \mathrm{m}$ pore size, VWR, Dietikon, Switzerland). A solvent blank was prepared in the same manner to correct for false positive results and additionally, pooled quality control (QC) samples were prepared by mixing $30 \mu \mathrm{L}$ of each final filtrate.

\subsection{UHPLC-QTOF MS analysis}

Hair samples were analyzed in randomized order to minimize effects of potential varying instrument performances over time. LC-MS analysis of hair samples was performed as described in detail elsewhere. ${ }^{21}$ Briefly, a Thermo Fischer Ultimate 3000 UHPLC system (Thermo Fischer Scientific, San Jose, CA) coupled to a high-resolution (HR) time-of-flight (TOF) instrument (TripleTOF 6600, Sciex, Concord, Ontario, Canada) was used in combination with reversed-phase (Waters XSelect HSST RP-C18 $(150 \mathrm{~mm} \times 2.1 \mathrm{~mm}$ i.d.; $2.5 \mu \mathrm{m}$ particle size; Waters (Baden-Daettwil, Switzerland)) and hydrophilic interaction liquid chromatography (Merck SeQuant ZIC HILIC column, $150 \mathrm{~mm} \times 2.1 \mathrm{~mm}, 3.5 \mu \mathrm{m}$ particle size). Gradient elution was performed after injection of $5 \mu \mathrm{L}$ sample with the following mobile phases: mobile phase A (10 mM ammonium formate in $\mathrm{H}_{2} \mathrm{O}$ containing $0.1 \%$ (v/v) formic acid), mobile phase $\mathrm{B}(\mathrm{MeOH}$ containing $0.1 \%(\mathrm{v} / \mathrm{v})$ formic acid), mobile phase $\mathrm{C}$ (25 $\mathrm{mM}$ ammonium acetate containing $0.1 \%(\mathrm{v} / \mathrm{v})$ acetic acid in $\mathrm{H}_{2} \mathrm{O}$ ) and mobile phase D (ACN containing $0.1 \%$ $(\mathrm{v} / \mathrm{v})$ acetic acid). Gradient elution on the reversed-phase column was as follows: $1 \mathrm{~min} 100 \% \mathrm{~A}$; 1-15 min increase to $100 \% \mathrm{~B}$, decrease to start conditions after $18 \mathrm{~min}$ and re-equi- libration for $2 \mathrm{~min}$. Furthermore, gradient conditions on the HILIC column were: $1 \mathrm{~min} 95 \% \mathrm{D}$; $1-10 \mathrm{~min}$ decrease to $40 \%$ $\mathrm{D} ; 10-12 \mathrm{~min}$ decrease to $10 \% \mathrm{D}$ and held for $1 \mathrm{~min}$; and increase to start conditions and re-equilibration for $4 \mathrm{~min}$. Acquisition of HR mass spectra (MS) and tandem mass spectrometry (MS/MS) data for each sample was achieved using TOF-MS only and information-dependent acquisition (IDA) in positive (HSST pos and HILIC pos) and negative (HSST neg and HILIC neg) electrospray ionization (ESI) mode. The MS analysis was performed with a DuoSpray ion source at a resolving power (full width at half maximum (fwhm) at $\mathrm{m} / \mathrm{z} 400$ ) of 30000 in MS and 30000 in MS2 (HR mode) or 15000 (highsensitivity mode). Automatic calibration was performed every five samples in positive ionization mode using an atmospheric-pressure chemical ionization (APCI) positive calibration solution (Sciex, Concord, Ontario, Canada) and every three samples in negative ionization mode using an APCI negative calibration solution (Sciex).

TOF-MS scans were performed over a mass range of $\mathrm{m} / \mathrm{z} 50$ to $m / z 1000$, with an accumulation time of $100 \mathrm{~ms}$ and a collision energy (CE) of $5 \mathrm{eV}$. IDA scans were performed with an accumulation time of $100 \mathrm{~ms}$ and a CE of $35 \mathrm{eV}$ with a CE spread of $15 \mathrm{eV}$ over a mass range of $\mathrm{m} / \mathrm{z} 50$ to $\mathrm{m} / \mathrm{z} 1000$. Further criteria for IDA experiments were as follows: dynamic background subtraction on the four most intense ions; intensity threshold above 100 counts per second (cps); exclusion time of $5 \mathrm{~s}$ (half peak width) after two occurrences in high sensitivity mode. Data acquisition of MS parameters was controlled by Analyst TF software 1.7 (Sciex, Concord, Ontario, Canada).

At the sequence beginning, blanks and pooled QC samples were injected to allow instrument equilibration. Pooled QC samples as a measure for stability of the analytical system ${ }^{22}$ were injected every 5 samples. Additionally, to check for reproducibility of the data and retention time (RT) shifts, a system suitability test (SST) prepared according to Steuer et al. ${ }^{23}$ was measured every tenth sample. Analyte peak areas of SST compounds and potential identified markers were obtained by targeted peak area integration of precursor ions from TOF-MS data using MultiQuant V 2.1 (Sciex, Concord, Ontario, Canada).

\subsection{Data processing, feature selection and statistical evaluation}

For the evaluation of metabolites with constant concentrations (in the following referred to as "stable") and altered metabolites between untreated and bleached hair samples, data-preprocessing, alignment, deconvolution, peak-picking, initial data normalization and filtering of TOF-MS data was performed in Progenesis QI (Waters Corp, Milford, USA). Data from IDA scans were incorporated for improved identification only. A pooled QC sample was chosen after automatic processing as an alignment reference. Peak-picking parameters were set as follows: automatic sensitivity method; sensitivity value 4; no minimum peak width; no retention time limits; ion species: $[\mathrm{M}+\mathrm{H}]^{+},[\mathrm{M}+2 \mathrm{H}]^{2+},\left[\mathrm{M}+\mathrm{H}-\mathrm{H}_{2} \mathrm{O}\right]^{+},\left[\mathrm{M}+\mathrm{NH}_{4}\right]^{+}$, 
$[\mathrm{M}+\mathrm{Na}]^{+},[\mathrm{M}+2 \mathrm{Na}]^{2+},[\mathrm{M}-\mathrm{H}]^{-},[\mathrm{M}-2 \mathrm{H}]^{-},\left[\mathrm{M}-\mathrm{H}_{2} \mathrm{O}-\mathrm{H}\right]^{-}$, $[\mathrm{M}+\mathrm{Na}-2 \mathrm{H}]^{-},[\mathrm{M}+\mathrm{FA}-\mathrm{H}]^{-}$. Data sets from HSST pos, HSST neg, HILIC pos and HILIC neg were filtered for features with an abundance $>1000$ cps in positive and $>500$ cps in negative ionization mode and available MS/MS spectra. All samples were finally normalized by the respective hair sample weight.

Further feature selection and statistical analyses were performed in MetaboAnalyst $4.0^{24}$ and GraphPad Prism 7 (GraphPad Software, San Diego, CA. USA). Prior to statistical analyses in MetaboAnalyst, data was auto-scaled (mean-centered and divided by the standard deviation of each variable). Principal component analysis (PCA) was applied for first visualization of the data. To search for changed metabolites, volcano plots were applied with the following settings: Wilcoxon matched-pairs test $(p<0.01$, false discovery rate (FDR) adjustment); fold-change analysis (threshold 2; significant paired count threshold 75\%). The average loss of features in treated hair samples was calculated pairwise in percent with feature abundance in untreated hair samples set to $100 \%$. Furthermore, GraphPad Prism 7.0 (GraphPad Software, San Diego, CA, USA) was used to generate receiver operating characteristic (ROC) curves.

\subsection{Compound identification}

Features of interest were searched against different databases on the MS and MS/MS level: the national institute of standards and technologies (NIST), ${ }^{25}$ the human metabolome database (HMDB), ${ }^{26}$ the online databases METLIN $^{27}$ and Lipidblast ${ }^{28}$ and after calculation of the elemental composition in Progenesis QI as well as against an in-house database in PeakView V 2.2 (Sciex). Identity was confirmed by matching library search results, accurate precursor masses and fragment ions as well as RT to reference standards, if available. Identification results were classified to four identification confidence levels as suggested by the metabolomics standards initiative (MSI). ${ }^{29}$

\section{Results and discussion}

\subsection{Bleaching procedure}

To study the changes between cosmetically untreated and oxidatively treated hair samples, genuine hair samples were bleached in vitro. For this, we applied a concentration of $9 \%$ $\mathrm{H}_{2} \mathrm{O}_{2}$ during $30 \mathrm{~min}$ as this is to be expected to definitely produce an effect. In typical bleaching or hair dyeing products, $\mathrm{H}_{2} \mathrm{O}_{2}$ concentrations range from $2 \%$ to $12 \%$. $^{30,31}$ The $\mathrm{H}_{2} \mathrm{O}_{2}$ concentration chosen within our study therefore lies in the upper range. These bleaching conditions do not only lead to a visible brightening of the hair but also the hair structure carries away a severe damage as can already be seen by microscopic observation. ${ }^{19,32}$ This might also facilitate the leakage and the extraction from the hair matrix. Nevertheless, it is to be expected that individuals adulterating their hair on purpose with the goal of a negative hair testing result would accept and encourage a considerably high hair damage.

\subsection{Analytical procedure}

Screening for potential differing metabolites due to oxidative hair treatment was performed with a previous evaluated LC-HRMS method. ${ }^{14,20}$ To avoid errors resulting from varying instrument performances over time, all samples were analyzed in randomized order and direct comparison of sample results was only performed within one batch. The analytical performance and the reproducibility of the batch analyses were monitored by deviation of RT and peak areas in SST and pooled QC samples. Mean coefficient of variations $(\mathrm{CV})$ of peak areas were determined for all SST compounds and were $7.8 \pm 2.9 \%$ (HSST pos), $11 \pm 2.3 \%$ (HSST neg), $14 \pm 5.3 \%$ (HILIC pos) and $21 \pm$ $4.8 \%$ (HILIC neg). CV's of QC samples were $<30 \%$ for the majority of the features $(>75 \%)$, as is generally recommended for the assessment of the data quality. ${ }^{22}$ Hence, all experiments showed good stability of the data over the analytical runs and were therefore used for further data analysis.

\subsection{Feature selection}

Progenesis QI was used for peak picking and data alignment. Compared to e.g. blood or urine samples, expected concentrations of exo- and endogenous compounds in hair samples are generally lower. ${ }^{1}$ To avoid missing compounds, the applied sensitivity value in Progenesis QI for peak-picking was optimized prior to further data analysis (data not shown). Increasing the sensitivity value lead to an increased number of detected compound ions. Even though this in turn also increased the background noise, it was accepted for this initial proof-of-concept study in order to detect as many potentially interesting candidates as possible. Processing in Progenesis QI initially lead to the detection of a maximum of 23000 compound ions. To allow for unambiguous identification, data was filtered for features (defined by RT and $\mathrm{m} / \mathrm{z}$ ) with MS/MS spectra only. Additionally, features with an abundance $<1000 \mathrm{cps}$ (in positive ionization) and $<500 \mathrm{cps}$ (in negative ionization) were removed due to their high CV's and low reproducibility.

For metabolites affected by cosmetic treatment, first visualization of the data by PCA analysis already showed clear separation of both groups in all four data sets as shown in Fig. 1. Moreover, volcano plots were applied to filter for features of significant interest. A significant paired count threshold of $75 \%$ was applied meaning that a minimum of $75 \%$ of the samples needed a consistent change above the given fold-change threshold. A total of 1053 (HSST pos), 903 (HSST neg), 1148 (HILIC pos) and 2005 (HILIC neg) features fulfilled the criteria and showed significant changes between untreated and bleached hair samples. The average loss of features across samples in percent can be found in Table 1.

To screen for metabolites remaining stable after treatment and between subjects, further filtering for features with a $\mathrm{CV}$ $<30 \%$ only was conducted. Additionally, a fold-change threshold $\leq 1.2$ and significance level with $p \geq 0.05$ had to be fulfilled. In total, 126 (HSST pos), 61 (HSST neg), 220 (HILIC pos) and 35 (HILIC neg) stable features were detected. These are low numbers compared to the number of total compound ions detected, assuming that the vast majority of hair metab- 

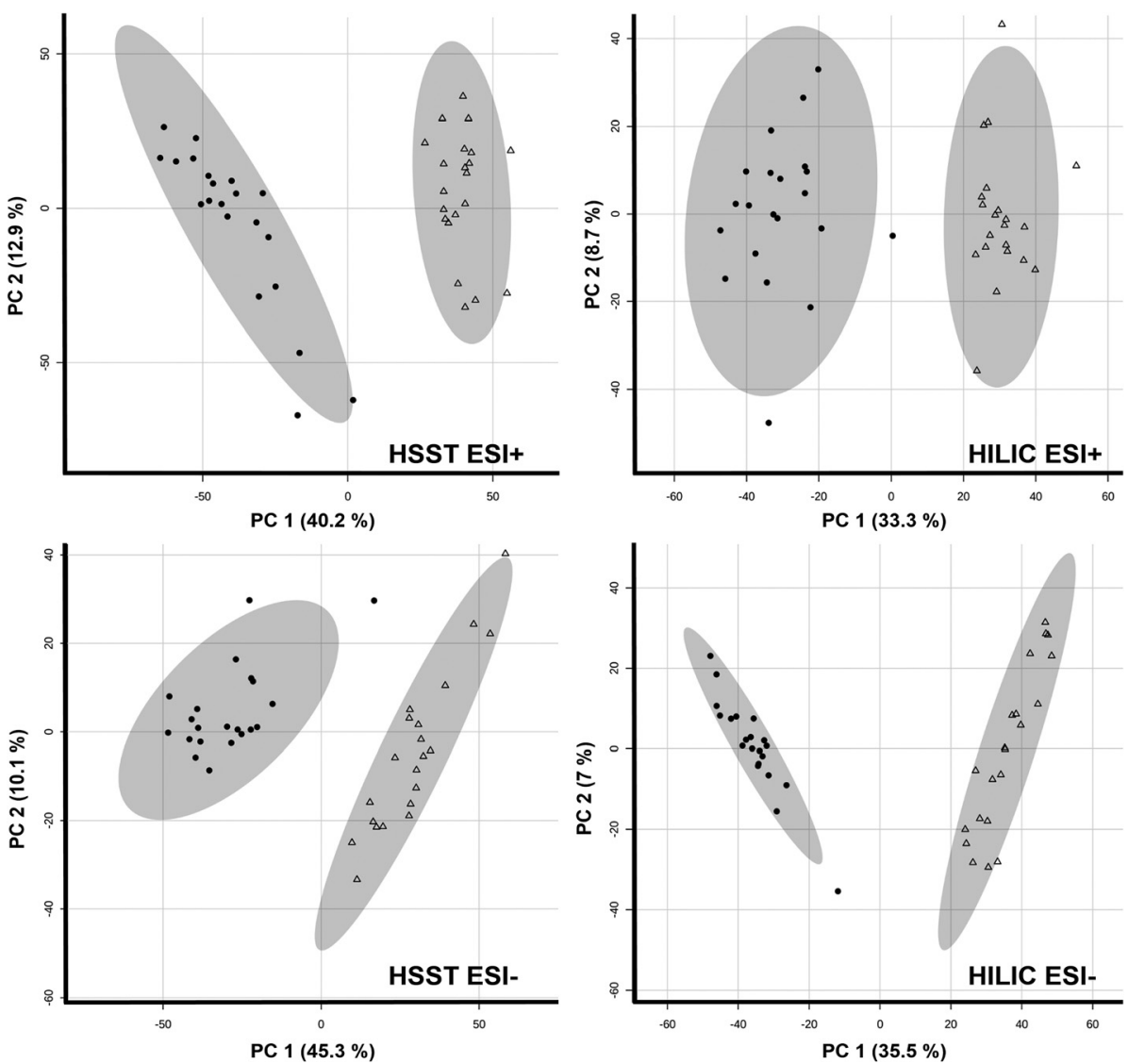

Fig. 1 Principal component analyses (PCA) of untreated (triangle) and treated $\left(9 \% \mathrm{H}_{2} \mathrm{O}_{2}, 30\right.$ min; radial form) hair samples ( $n=21$ each).

olites is affected through the quite aggressive oxidative bleaching procedure. To avoid missing of important stable features through the applied MS/MS filter, the same processing has been performed including also features without MS/MS data. The number of stable features however only increased minimally (e.g. from 126 to 144 features in HSST pos mode).

\subsection{Feature identification}

Hit lists of features were manually checked for positive hits. In total, 69 altered metabolites could be identified, thereof 43 metabolites with an identification level $1^{29}$ after comparison of MS/MS spectra and RT to a reference standard as summarized in Table 1. For the purpose of the study, only metabolites with an identification level of 2 and better were considered in this study as unambiguous identification is necessary for their implementation into routine analysis. No stable metabolites could be identified with sufficient confidence (level 2 or better). Compounds resulting clearly from exogenous origin (e.g. caffeine, dexpanthenol) were not further investigated as inter-individual differences are unpredictable and marker suitability is considered as low. Some compounds were detected on both columns and/or different ionization modes and are listed as a single compound in Table 1. If insufficient chromatographic separation hindered an exact identification, the MSI level was assigned to level 2. From the hit lists of sig- nificantly altered features, a high number of features could be assigned to the compound class of peptides (see also Table $\mathrm{S} 1 \dagger)$. As the protein content of hair is very high, ${ }^{33}$ this supports the hypothesis of an enhanced degradation and break-down of proteins from the hair matrix, thus extending the feature hit list.

As discussed in detail in a previous publication, the "hair metabolome" is still insufficiently described. ${ }^{20}$ Thus, many metabolites which might be hair specific might not be represented within the different databases and are therefore unamenable to identification. The majority of identified metabolites was found to be decreased in concentration or even totally degraded through the bleaching process. Higher levels due to bleaching were only observed for adenosine and its derivatives. Neo-formation or the detection of direct oxidation products was only observed for melanin degradation products and oxidation products of cysteine and cystine. The difficulty to identify new oxidation products is based on the fact that spectral databases used for metabolomics deal with endogenous compounds altered under physiological conditions only. The bleaching process however is an "artificial" influence on the endogenous metabolites which produces oxidation products that might not be present under physiological conditions and have therefore not (yet) been described and studied. Hence, they might rarely be present in currently avail- 


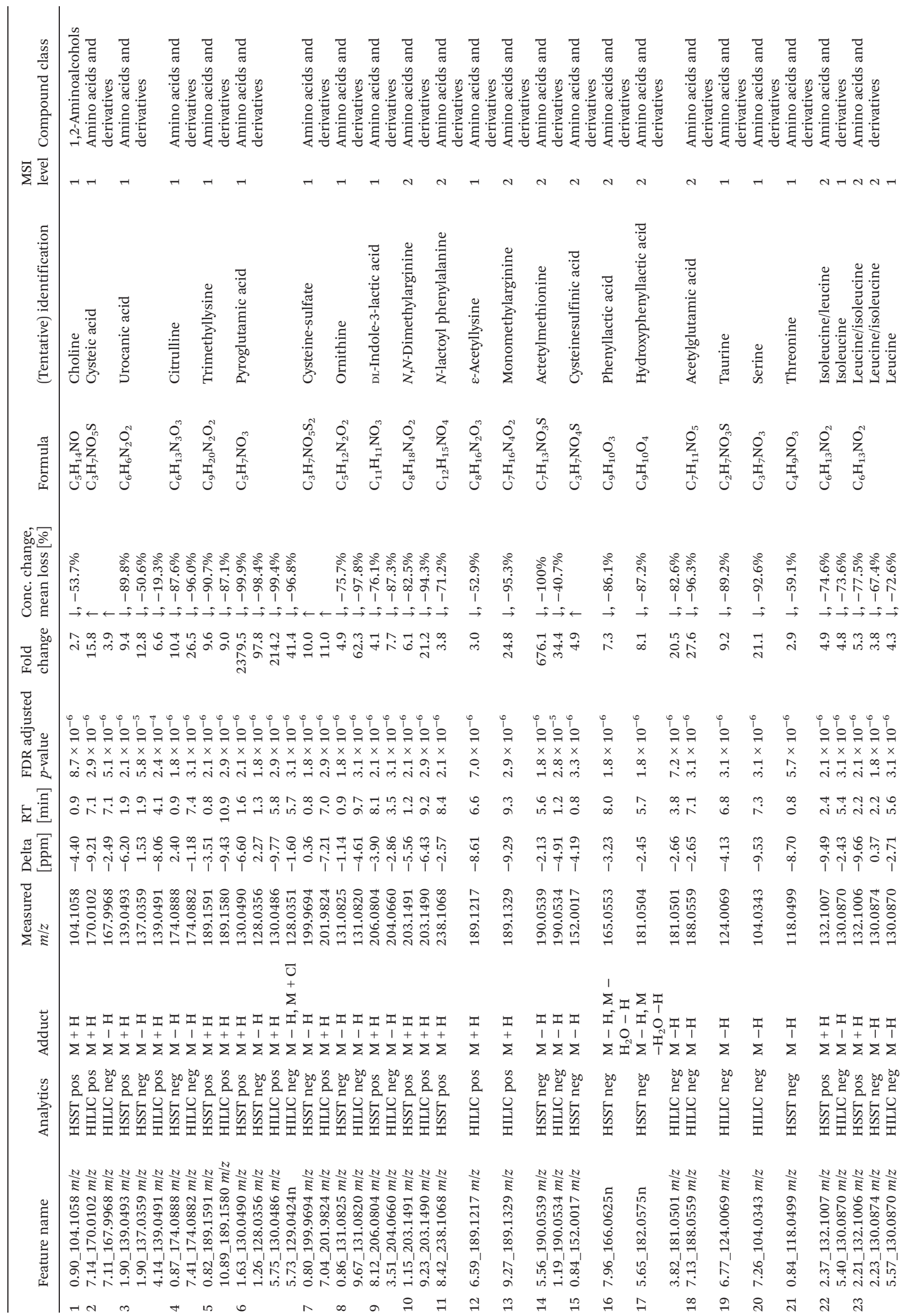




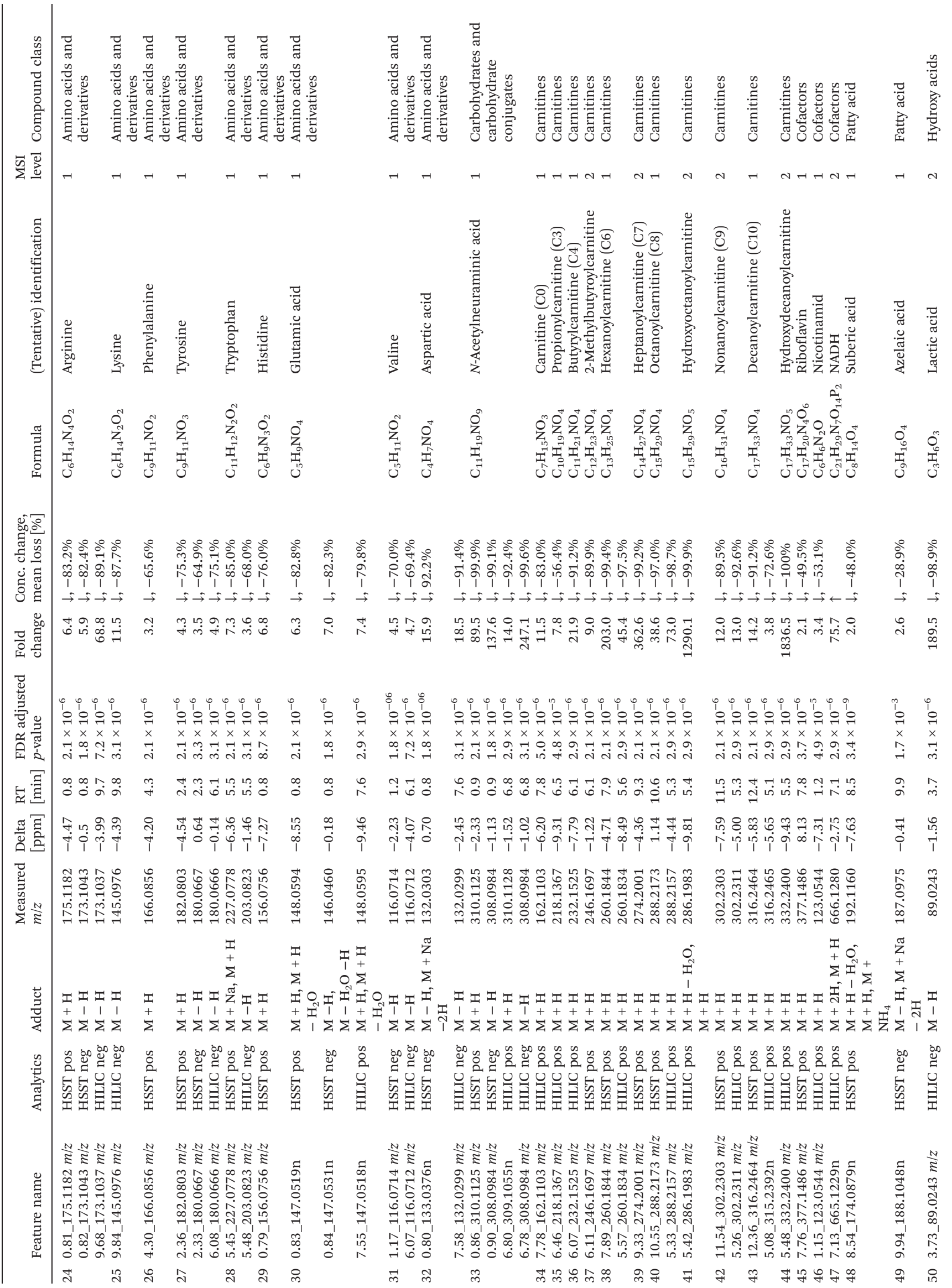




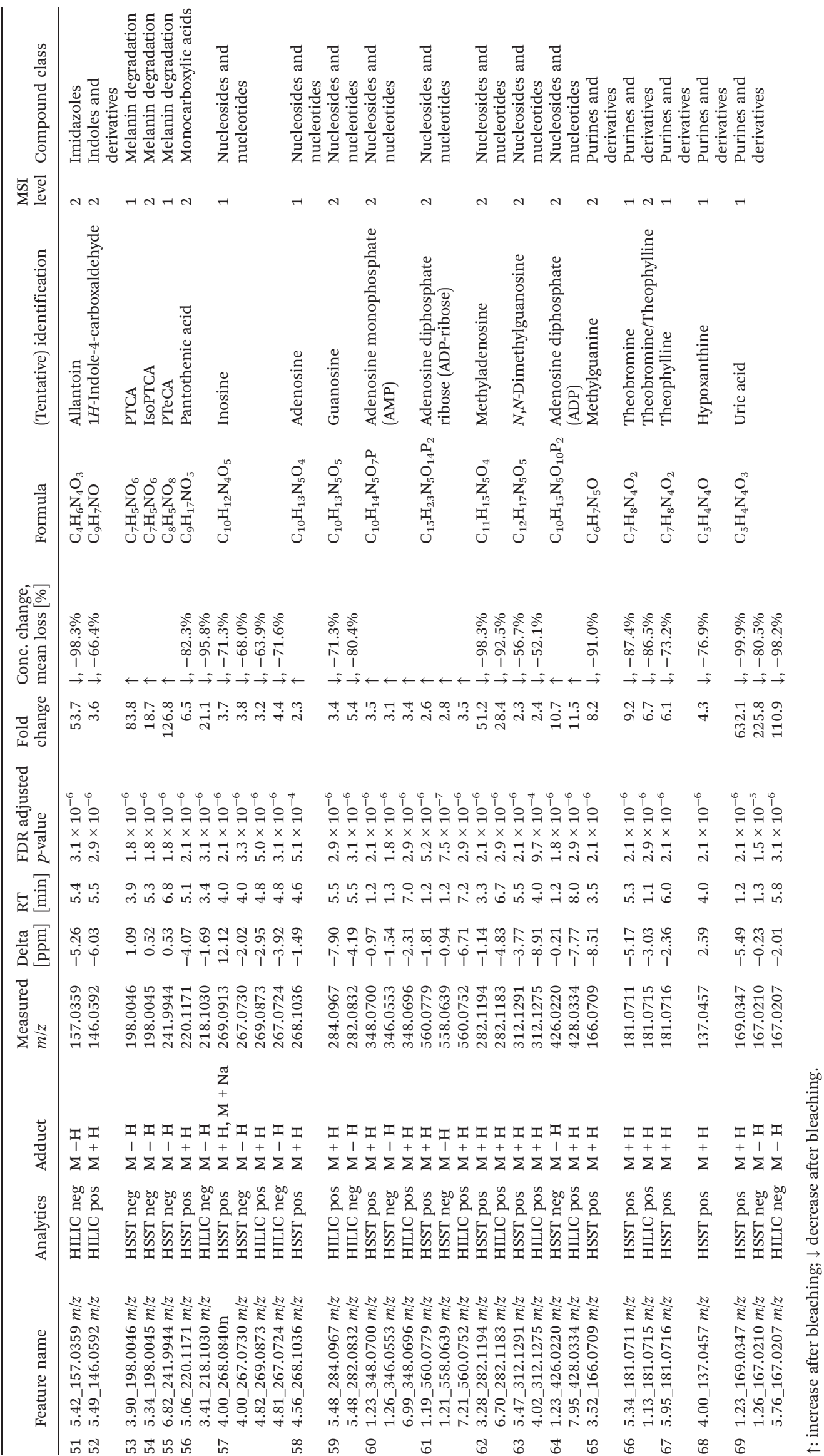


able databases. In addition, follow-up reactions of oxidation products or multiple oxidations are difficult to predict and to identify in a complex sample. Even after identification, such oxidation products might rarely be available as reference material for routine analysis. Therefore, an intensified search for further oxidation products was omitted and focus applied on the (high) number of identified compounds.

\subsection{Marker interpretation}

In general, a high number of compounds was found to be significantly altered between untreated and bleached hair samples. Altogether, with this untargeted workflow, we found compound changes in all directions: increase, decrease, total degradation and neo-formation (see Table 1). To act as a potential biomarker, a compound should ideally be usable in qualitative screening procedures without the time-intensive and complex establishment of a cut-off value. This is only true for newly formed (oxidation) products that are missing in authentic hair samples. Total degradation of endogenous compounds could be useful, but might also depend on the sensitivity of the method. The used Q-TOF method was selected for its broad screening and identification properties, but lacks sensitivity compared to typical quantification instruments such as triple quadrupols in multiple reaction monitoring mode. Compounds that are undetectable in the current setting might still be measurable in low amounts when more sensitive methods are applied. For metabolites that are not solely formed through adulteration but only altered in abundance, high inter-individual concentration differences in the metabolome due to lifestyle habits or diseases ('endogenous variances') and/or due to the complex nature of hair samples ('exogenous variances') are expected, even without oxidative treatments. Therefore, at best, markers from different meta- bolic pathways should be screened for. Furthermore, additional strategies should be considered, such as formation of metabolite ratios or normalization to endogenous metabolites unaffected by treatment and homogenously distributed among the population. Compound classes predominantly identified as significantly altered after bleaching include melanin degradation products, carnitines, amino acids and derivatives, as well as purines, nucleosides and derivatives and will be discussed in the following subchapters.

3.5.1 Melanin degradation products. Melanin, composed of eumelanin and pheomelanin, is responsible for the pigmentation of natural human hair. ${ }^{34}$ Through oxidation processes or UVA-induced photoaging, it can be degraded by oxidation and/or cross-linking of its indole-moieties. ${ }^{35-37}$ In line with previous work, ${ }^{13,14}$ the applied untargeted workflow allowed for the detection of the eumelanin degradation products PTCA, $1 H$-pyrrole-2,3,4-tricarboxylic acid (isoPTCA) and PTeCA either as significantly increased (PTCA, isoPTCA) or as newlyformed (PTeCA) after bleaching. ${ }^{13,14}$ This consistence proofs the general applicability of the current untargeted workflow to detect potential markers for oxidative hair treatment.

3.5.2 Carnitines. As shown in Table 1, carnitine and acyl carnitines were predominantly identified as significantly altered after bleaching. carnitine (C0) and acylcarnitines are essential for fatty acid metabolism by serving as carriers of long chain fatty acids into mitochondria for subsequent $\beta$-oxidation. ${ }^{38}$ So far, information on presence, actual expected concentrations and function of (acyl)carnitines in hair are scarce. L-Carnitine-L-tartrate was described as hair growth promoting in vitro ${ }^{39}$ and usage of propionylcarnitine (C3) in liposome-based formulations to prevent and treat hair loss was discussed. ${ }^{40}$ An extracted ion chromatogram of carnitines before and after bleaching can be found in Fig. 2. Detailed

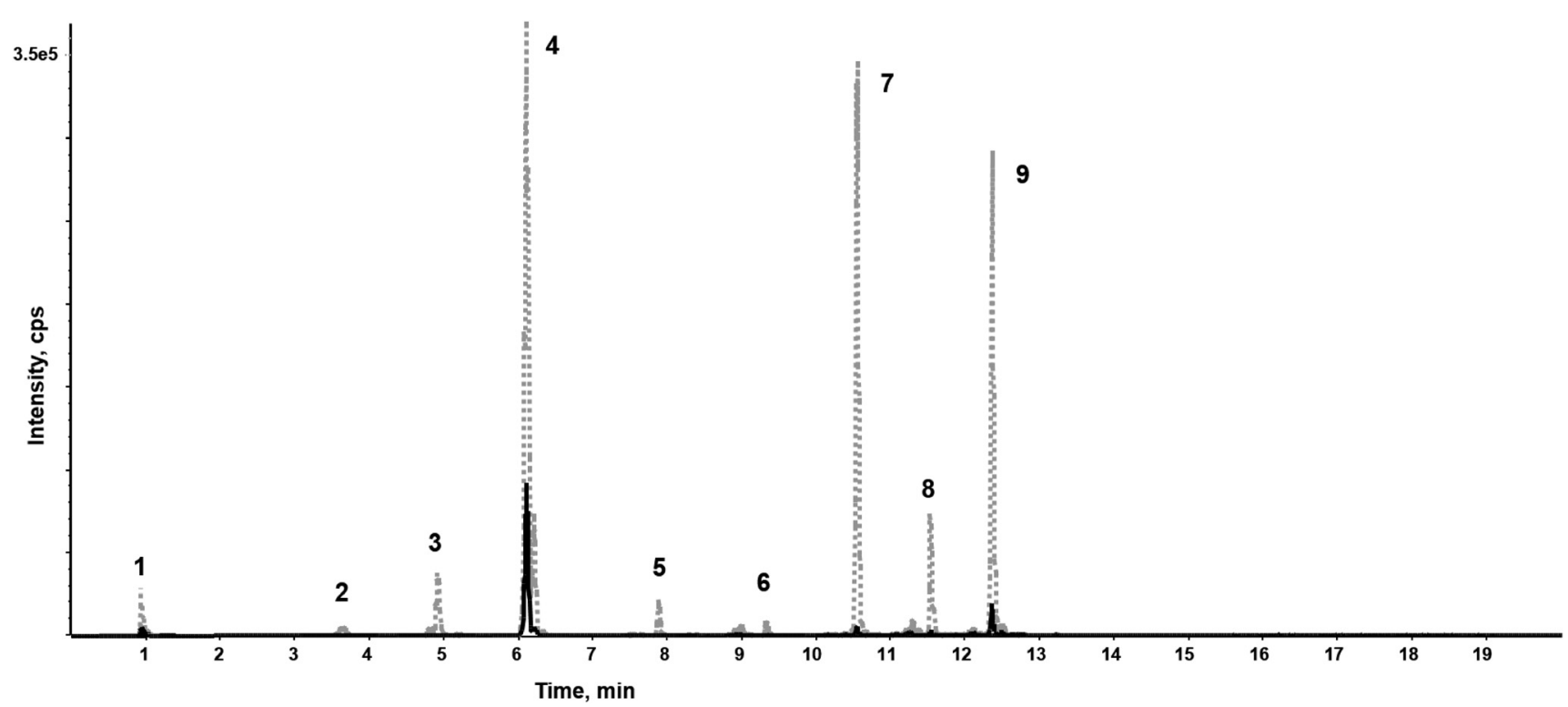

Fig. 2 Extracted ion chromatograms (XIC) of identified carnitines before (grey, dotted line) and after bleaching (black, solid line) in a representative hair sample, measured on a HSST column in positive ionization mode. 1: carnitine; 2: propionylcarnitine; 3: butyrylcarnitine; 4: 2-methylbutyroylcarnitine; 5 : hexanoylcarnitine; 6 : heptanoylcarnitine; 7: octanoylcarnitine; 8: nonanoylcarnitine; 9: decanoylcarnitine. 

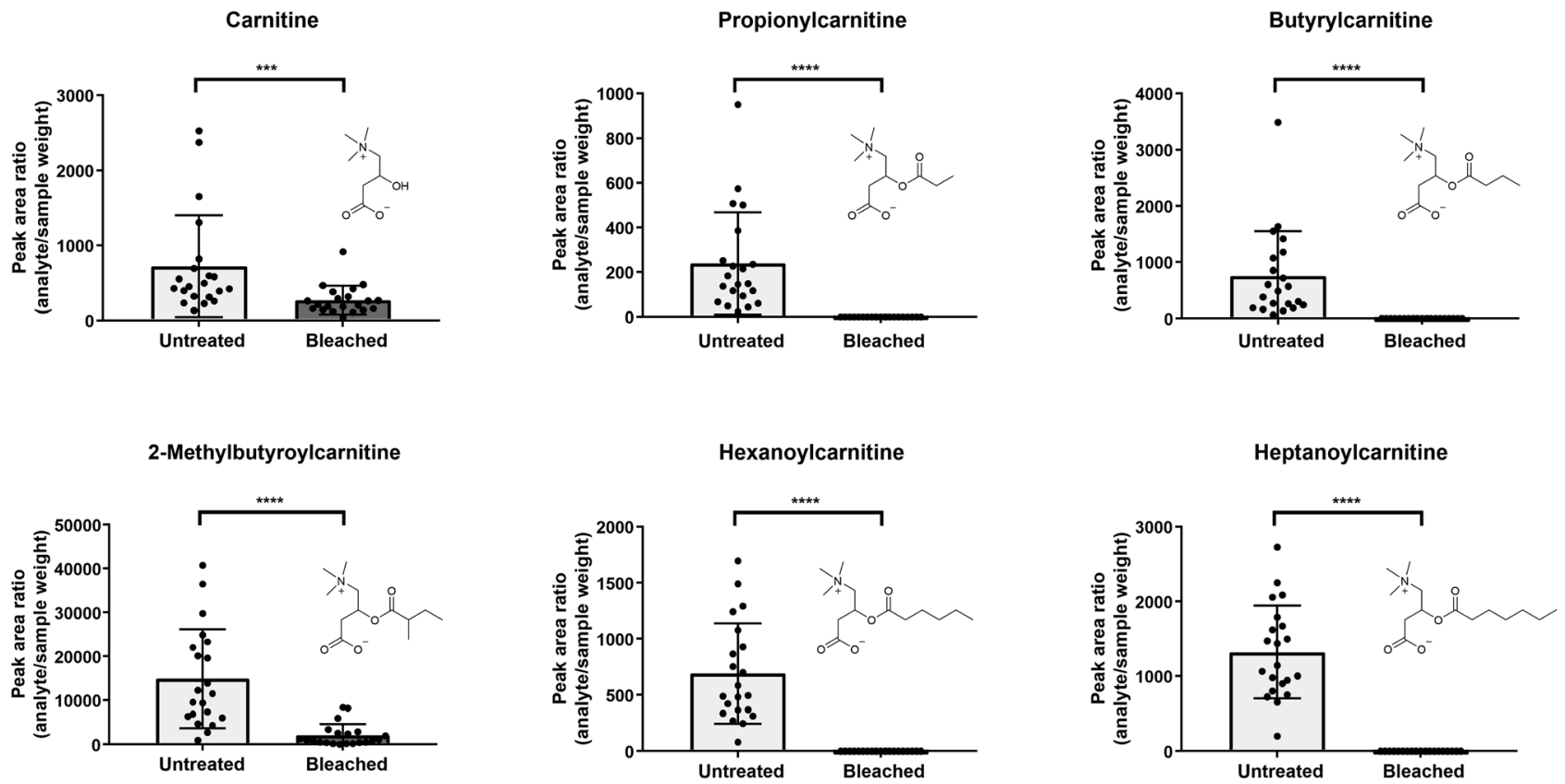

Octanoylcarnitine

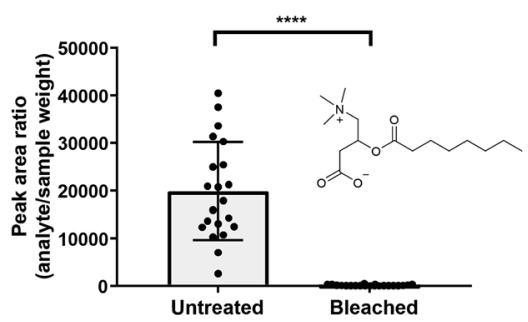

Nonanoylcarnitine

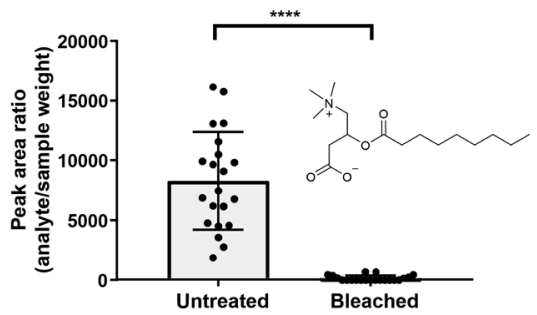

Decanoylcarnitine

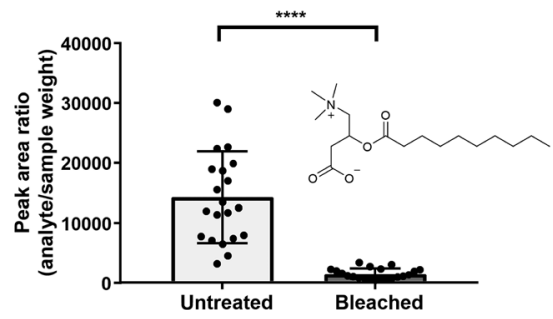

Fig. 3 Comparison of carnitines between untreated (light grey) and bleached (dark grey) hair samples (analysis on HSST in positive ionization mode). Displayed are analyte peak area ratios (analyte area/sample weight); error bars present standard deviations, $n=21$ each. Statistical analysis with Wilcoxon test; $p<0.001{ }^{(* *)}, p<0.0001(* * *)$.

comparisons of carnitines in untreated and treated samples are depicted in Fig. 3. Acetylcarnitine (C2) could only be identified after targeted evaluation of HILIC pos data, but not with the standard untargeted workflow. It is therefore not listed as identified marker but should of course be included in further studies, as in line with the other acyl carnitines, a significant decrease in treated hair samples was observed.

As depicted in Fig. 3, five out of 9 detected carnitines were degraded after bleaching. Mainly low abundant acylcarnitines were undetectable, assuming that complete analyte degradation after bleaching might be dependent on initial metabolite concentrations and/or instrument sensitivity. If increased lipophilicity causes higher incorporation into the hair matrix, ${ }^{1}$ increasing chain length of the acyl moiety should result in higher levels in bleached hair samples. Yet, this correlation could not be confirmed in our study.

Selecting various metabolites over one single marker can increase confidence of the analytical findings. Kluge et al. investigated the suitability of endogenous biomolecules as val- idity parameters for the detection of manipulated urine samples. ${ }^{41}$ Samples were considered as manipulated if less than six out of 10 endogenous biomolecules (therefrom six acylcarnitines: C3, butyrylcarnitine (C4), isovalerylcarnitine, hexanoylcarnitine (C6), heptanoylcarnitine (C7) and octanoylcarnitine (C8)) were identified by an untargeted LC-MS/MS screening method. ${ }^{41}$ In line, we propose, that undetectability of highly abundant carnitines such as e.g. $\mathrm{C} 8$ in hair would highlight a sample as suspicious. C4 and C8 especially showed high prevalences with detection rates $>98 \%$ in authentic urine samples. ${ }^{41}$ However, similar comprehensive data on expected prevalence of carnitines in hair samples is still missing.

3.5.3 Amino acids and amino acid derivatives. Amino acids present the building blocks of proteins. Thus, besides incorporation from the blood stream, (proteinogenic) amino acids form the major element of the hair structure, the keratin protein. ${ }^{42}$ Derivatives of amino acids can be formed within metabolic pathways (e.g. enzymatically or non-enzymatically) and are subsequently incorporated into the hair matrix. 
Additionally, due to its constant external exposure, amino acids can be chemically converted through influences such as sunlight exposure, hair straightening or hair bleaching. ${ }^{43}$ Within our study, a high number of amino acids, amino acid derivatives and peptides could be identified (see Table 1 and Table S1†). Except for cysteic acid, cysteinesulfate and cysteine sulfininic acid which were significantly elevated, bleaching lead to a general decrease and degradation of amino acids and derivatives. This is not surprising as the protein structure is severely damaged through oxidative treatments and amino acids are rapidly attacked by untargeted oxidative reactions.

Cysteine is predominantly present in the keratin structure. Sulphur groups of cysteine are oxidized to cystine forming disulphide bonds in keratin and giving strength to the hair structure. The sulphur atoms of cysteine and cystine are sensitive to oxidative processes which leads to the formation of cysteic acid. As also shown by other authors, cysteic acid is significantly increased in bleached hair samples and is widely monitored as a marker for bleach-induced hair damage. ${ }^{18}$ Moreover, the untargeted workflow allowed for the additional identification of cysteinesulfate and cysteinesulfinic acid as oxidation products of cystine and cysteine respectively. Even though all three cysteine derivatives were formed during bleaching, they were also detectable in untreated hair samples. On the contrary, despite the intense bleaching, amino acids and derivatives that decreased in treated samples still remained detectable in bleached hair samples, as exemplarily depicted for a selection of amino acids in Fig. 4. We therefore tested different biomarker ratios of elevated and lowered amino acids for their ability to increase the discrimination power and to avoid the need for defined cut-off values. Ratios of tyrosine/cysteic acid and tryptophan/cysteic acid in untreated and treated hair samples are given in Fig. 5A. ROC curves were applied to illustrate the discrimination power of biomarker ratios. With calculated area under the curve (AUC) values approaching one, the prediction power of a biomarker or biomarker ratio in terms of specificity and sensitivity increases. In contrast to the single amino acids, complete discrimination of untreated and treated hair samples was possible by biomarker ratios. That means, if the ratio of tyrosine/ cysteic acid falls below a certain threshold, this would denote an authentic hair sample as suspicious. Higher numbers of hair samples followed by amino acid (semi-) quantification will be necessary to confirm these initial findings prior to routine applications.

3.5.4 Purines, nucleosides and nucleotides and $\mathrm{N}$-acetylneuraminic acid. As summarized in Table 1, numerous purines, nucleosides and nucleotides were found in lowered concentrations after treatment. Interestingly, except for methyladenosine, signals of adenosine and adenosine containing metabolites increased after bleaching. Adenosine plays an important role as a building block for DNA or RNA and is involved in energy transfer and signal transduction. Furthermore, adenosine is described as hair growth promoting and thus used to treat hair loss as a result of androgenetic alopecia. $^{44,45}$ Possibly, due to its hair growth promoting role, adenosine might be present in hair samples in higher concentrations compared to e.g. guanosine. Notwithstanding the above assumption, a facilitated extraction from the bleachdamaged hair structure could explain its increase. Nevertheless, as adenosine containing hair products are widely available, a documentation of applied hair products should carefully be conducted at the time of sampling to avoid misinterpretation of false positive adenosine concentrations. In contrast to adenosine, guanosine decreased in hair samples after bleaching. Oxidation of guanosine to 8-oxo-7,8-dihydroguanosine (8-oxo-dG) is the most frequent oxidation product in DNA. $^{46}$ Compared to adenosine, guanosine might therefore likewise be more prone to bleaching processes. Biomarker ratios of decreasing guanosine and increasing adenosine were formed, but did not result in improved discrimination. Yet, use of ratios of adenosine/hypoxanthine and adenosine/ inosine was able to improve the differentiation of untreated and oxidatively treated hair samples as shown in Fig. 5B. These biomarker ratios could be promising for hair manipulation monitoring.

Overall, best markers identified within this subgroup were uric acid and $\mathrm{N}$-acetylneuraminic acid, as they were totally degraded. These results are comparable to studies by Steuer et al. who investigated the detection and marker suitability of endogenous biomolecules in urine samples adulterated with potassium nitrite. ${ }^{23}$ The authors were additionally able to
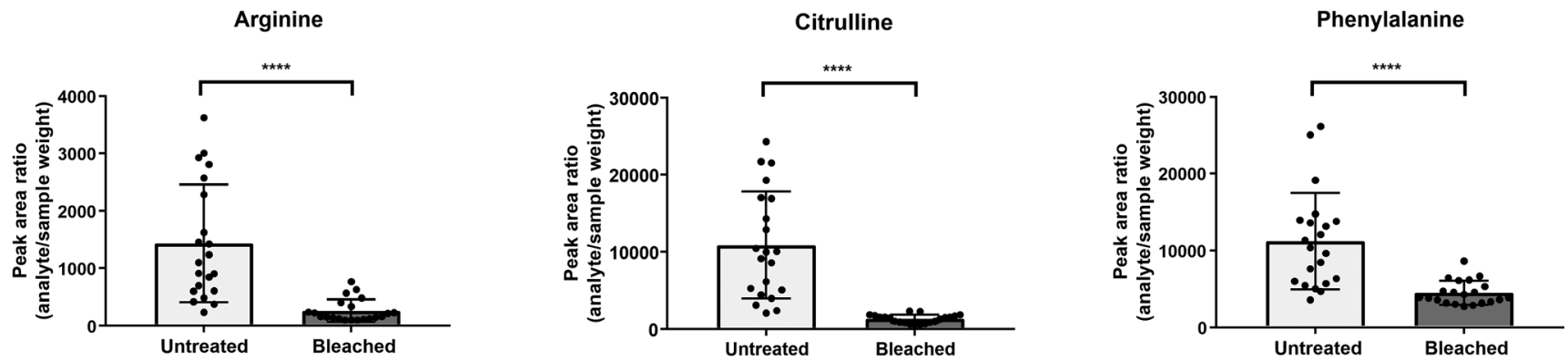

Fig. 4 Comparison of a selection of amino acids showing typically observed changes between untreated (light grey) and bleached (dark grey) hair samples. Displayed are analyte peak area ratios (analyte area/sample weight); error bars present standard deviations, $n=21$ each. Statistical analysis with Wilcoxon test; $p<0.0001(* * *)$. 
A)
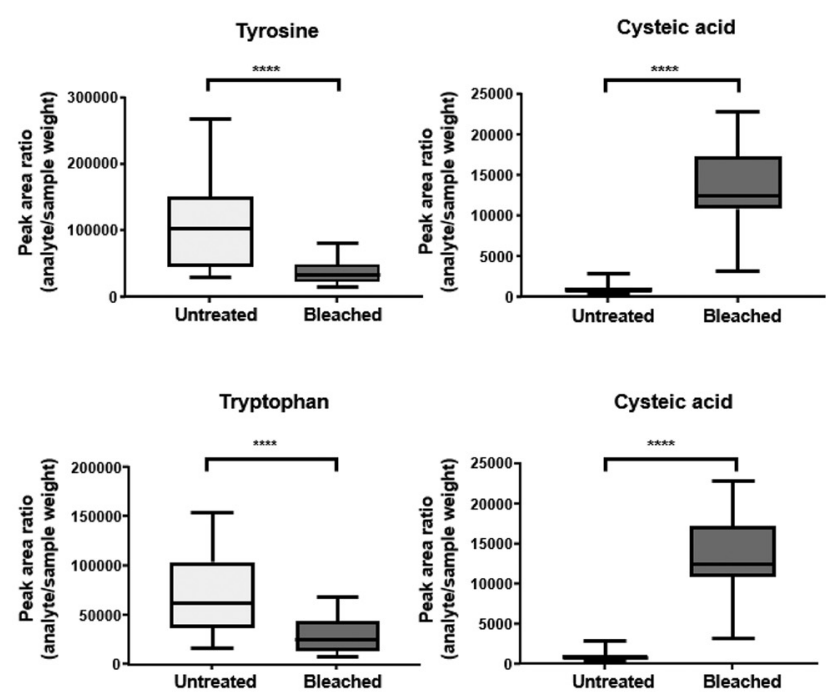

B)
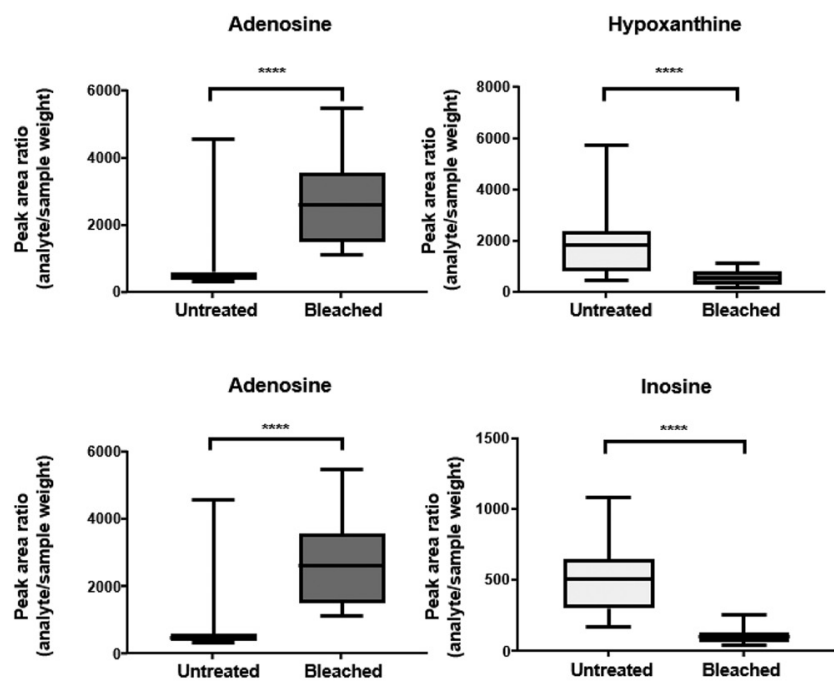
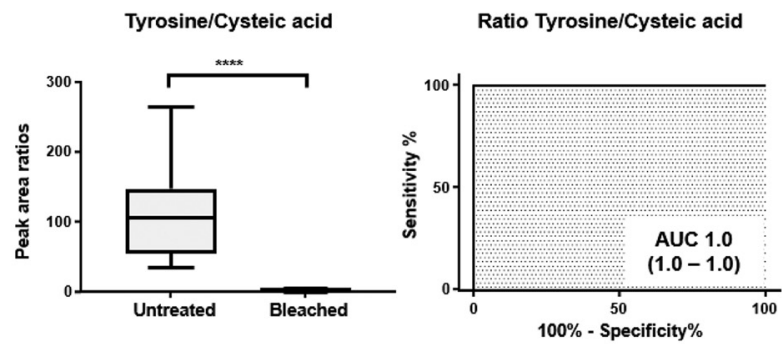

Tryptophan/Cysteic acid
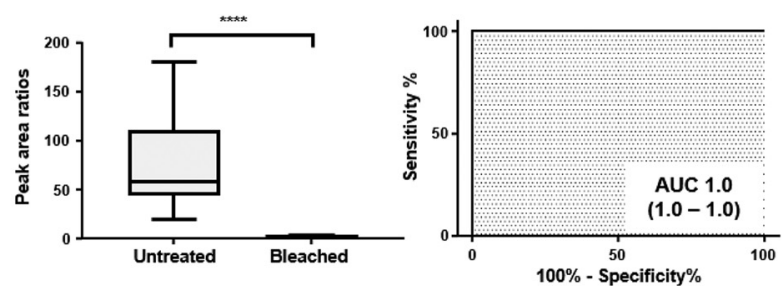

Ratio Tryptophan/Cysteic Acid

$00 \%$ - Specificity $\%$

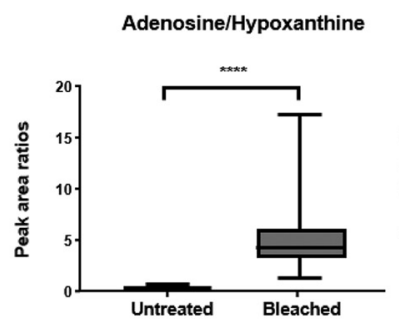

Ratio Adenosine/Hypoxanthine

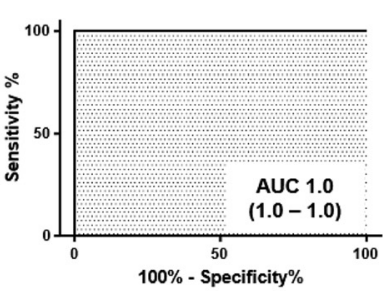

Adenosine/Inosine

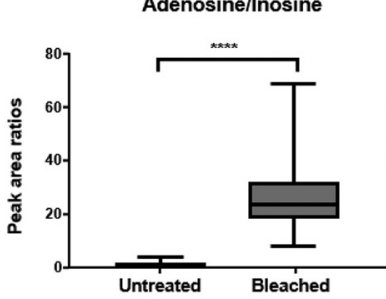

Ratio Adenosine/lnosine

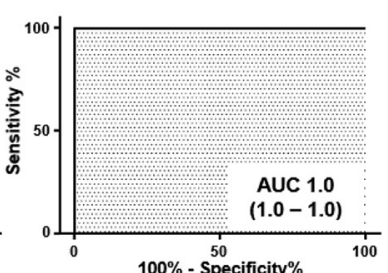

Fig. 5 Displayed are analyte peak area ratios (peak area/sample weight) and corresponding ratios thereof in untreated (light grey) and bleached (dark grey) hair samples in combination with receiver operating characteristic (ROC) curves of metabolite ratios (given are AUC and $95 \%$ confidence intervals). (A) HILIC neg (B) HSST pos. Whiskers of box plots represent minimum and maximum values, statistical analysis with Wilcoxon test: $p<$ $0.0001(* \star * *)$.

identify corresponding oxidation products of uric acid as 5-hydroxy-isourate and 5-hydroxy-2-oxo-4-ureido-2,5-dihydro$1 H$-imidazole-5-carboxylate, albeit in very low abundance. These oxidation products could not be identified in our study even after targeted data evaluation. Most likely, concentrations of these markers in hair are far below the sensitivity limit of the method.

3.5.5 Metabolites unaffected through bleaching. To omit tedious cut-off evalutions and investigation of baseline concentrations for a number of potentially markers, normalization to stable predefined hair markers would be a very promising approach. Such normalization markers need to fulfill the following criteria: homogenously present among hair samples, unaffected by endo- or exogenous influences and low interindividual variation. The untargeted nature of data acquisition applied in the present workflow should also allow the search for such markers. However, only a low number of metabolites was identified as stable between untreated and treated and between subjects. Unfortunately, reliable identification of these features with a sufficient identification level (level 2 and better) remained impossible until now. Not only for biomarker detection for oxidative hair treatments, but also for hair meta- 
bolomics in general, the detection of markers unaffected by external and internal influences would be a huge advancement. Leveling-out inter- or intra-individual effects would significantly increase the interpretation of hair metabolomic studies and should be followed up in future studies.

Finally, the herein used HRMS method is a highly complex, sophisticated and time-intensive method. Typical routine drug screenings are usually performed on triple quad instruments with only one chromatographic column and in positive ionization mode only. Therefore, our study focused on the identification of a broad range of potential biomarkers that could then be implemented individually into routine methods. Useful combinations of markers and/or marker ratios in terms of metabolite coverage and detection rates in general and sufficient analytical confidence in a routine setting (sensitive detection, analytical validity) allowing for reliable detection of adulteration attempts then have to be evaluated in routine methods. Altogether, the applicability of identified markers or marker ratios to real case samples from routine case work as well as their suitability to detect other adulteration attempts and hair treatments like e.g. permanent hair dyeing or thermal hair straightening should be investigated in future studies.

\section{Conclusions}

Using an untargeted hair metabolomics workflow, numerous potential markers and marker ratios for indirect detection of oxidative hair manipulation were identified. The variety of identified markers now offers the possibility to individually implement a biomarker selection into routine methods of different laboratories. Further studies with higher numbers of hair samples are needed to test the routine applicability in more sensitive targeted approaches and to determine general detection rates of endogenous biomolecules, selectivity and specificity. Furthermore, the applicability of identified markers to other cosmetic hair treatments should be studied to further improve the interpretation of hair testing results.

\section{Compliance with ethical standards}

All samples were analyzed in full conformance with Swiss laws (statement of Cantonal Ethics Board of the Canton of Zurich: BASEC-no. Req-2017-00946). According to Swissethics (Humanforschungsgesetz), no further ethical approval from the cantonal ethic commission is necessary if the research is not aiming to investigate diseases or functions of the human body as is the case in the current study.

\section{Conflicts of interest}

The authors declare that they have no conflict of interest.

\section{Acknowledgements}

The authors would like to express their gratitude to Emma Louise Kessler, MD for her generous legacy she donated to the Institute of Forensic Medicine at the University of Zurich, Switzerland for research purposes.

\section{References}

1 F. Pragst and M. A. Balikova, Clin. Chim. Acta, 2006, 370, 17-49.

2 C. Jurado, P. Kintz, M. Menendez and M. Repetto, Int. J. Leg. Med., 1997, 110, 159-163.

3 G. Skopp, L. Potsch and M. R. Moeller, Forensic Sci. Int., 1997, 84, 43-52.

4 I. Kerekes and M. Yegles, Ther. Drug Monit., 2013, 35, 527529.

5 J. Ettlinger, L. Kirchen and M. Yegles, Drug Test. Anal., 2014, 6(Suppl 1), 74-77.

6 T. M. Binz, M. R. Baumgartner and T. Kraemer, Forensic Sci. Int., 2014, 244, 20-24.

7 M. del Mar Ramirez Fernandez, S. M. R. Wille, V. Di Fazio and N. Samyn, Forensic Sci. Int., 2019, 297, 277-283.

8 L. Morini, A. Zucchella, A. Polettini, L. Politi and A. Groppi, Forensic Sci. Int., 2010, 198, 23-27.

9 N. Van Elsue and M. Yegles, Forensic Sci. Int., 2019, 297, 270-276.

10 S. Petzel-Witt, W. Pogoda, C. Wunder, A. Paulke, M. Schubert-Zsilavecz and S. W. Toennes, Drug Test. Anal., 2018, 10, 177-183.

11 S. Witt, C. Wunder, A. Paulke, M. A. Verhoff, M. SchubertZsilavecz and S. W. Toennes, Drug Test. Anal., 2016, 8, 826831.

12 D. Ammann, R. Becker, A. Kohl, J. Hanisch and I. Nehls, Forensic Sci. Int., 2014, 244, 30-35.

13 S. Petzel-Witt, S. I. Meier, M. Schubert-Zsilavecz and S. W. Toennes, Drug Test. Anal., 2018, 10, 768-773.

14 L. Eisenbeiss, T. M. Binz, M. R. Baumgartner, A. E. Steuer and T. Kraemer, Drug Test. Anal., 2020, 12, 230-238.

15 S. Luong, R. Shimmon, J. Hook and S. Fu, Anal. Bioanal. Chem., 2012, 403, 2057-2063.

16 S. Luong and S. Fu, Drug Test. Anal., 2014, 6, 277287.

17 W. B. Dunn, D. Broadhurst, P. Begley, E. Zelena, S. FrancisMcIntyre, N. Anderson, M. Brown, J. D. Knowles, A. Halsall, J. N. Haselden, A. W. Nicholls, I. D. Wilson, D. B. Kell, R. Goodacre and Human Serum Metabolome Consortium, Nat. Protoc., 2011, 6, 1060-1083.

18 K. M. Joo, A. R. Kim, S. N. Kim, B. M. Kim, H. K. Lee, S. Bae, J. H. Lee and K. M. Lim, Exp. Dermatol., 2016, 25, 729-731.

19 A. J. Grosvenor, S. Deb-Choudhury, P. G. Middlewood, A. Thomas, E. Lee, J. A. Vernon, J. L. Woods, C. Taylor, F. I. Bell and S. Clerens, Int. J. Cosmet. Sci., 2018, 40, 536548. 
20 L. Eisenbeiss, A. E. Steuer, T. M. Binz, M. R. Baumgartner and T. Kraemer, Anal. Bioanal. Chem., 2019, 411, 39633977.

21 M. I. Boxler, T. D. Schneider, T. Kraemer and A. E. Steuer, Drug Test. Anal., 2019, 11, 678-696.

22 H. G. Gika, G. A. Theodoridis, R. S. Plumb and I. D. Wilson, J. Pharm. Biomed. Anal., 2014, 87, 12-25.

23 A. E. Steuer, K. Arnold, T. D. Schneider, M. Poetzsch and T. Kraemer, Anal. Bioanal. Chem., 2017, 409, 6235-6244.

24 J. Chong, O. Soufan, C. Li, I. Caraus, S. Li, G. Bourque, D. S. Wishart and J. Xia, Nucleic Acids Res., 2018, 46, W486W494.

25 E. P. J. Linstrom and W. G. Mallard, NIST Chemistry WebBook, NIST Standard Reference Database Number 69, National Institute of Standards and Technology, Gaithersburg MD, 2001, p. 20899, DOI: 10.18434/T4D303, (retrieved November 8, 2018).

26 D. S. Wishart, D. Tzur, C. Knox, R. Eisner, A. C. Guo, N. Young, D. Cheng, K. Jewell, D. Arndt, S. Sawhney, C. Fung, L. Nikolai, M. Lewis, M. A. Coutouly, I. Forsythe, P. Tang, S. Shrivastava, K. Jeroncic, P. Stothard, G. Amegbey, D. Block, D. D. Hau, J. Wagner, J. Miniaci, M. Clements, M. Gebremedhin, N. Guo, Y. Zhang, G. E. Duggan, G. D. Macinnis, A. M. Weljie, R. Dowlatabadi, F. Bamforth, D. Clive, R. Greiner, L. Li, T. Marrie, B. D. Sykes, H. J. Vogel and L. Querengesser, Nucleic Acids Res., 2007, 35, D521-D526.

27 C. Guijas, J. R. Montenegro-Burke, X. Domingo-Almenara, A. Palermo, B. Warth, G. Hermann, G. Koellensperger, T. Huan, W. Uritboonthai, A. E. Aisporna, D. W. Wolan, M. E. Spilker, H. P. Benton and G. Siuzdak, Anal. Chem., 2018, 90, 3156-3164.

28 T. Kind, K. H. Liu, D. Y. Lee, B. DeFelice, J. K. Meissen and O. Fiehn, Nat. Methods, 2013, 10, 755-758.

29 L. W. Sumner, A. Amberg, D. Barrett, M. H. Beale, R. Beger, C. A. Daykin, T. W. Fan, O. Fiehn, R. Goodacre, J. L. Griffin, T. Hankemeier, N. Hardy, J. Harnly, R. Higashi, J. Kopka, A. N. Lane, J. C. Lindon, P. Marriott, A. W. Nicholls, M. D. Reily, J. J. Thaden and M. R. Viant, Metabolomics, 2007, 3, 211-221.
30 A. Guerra-Tapia and E. Gonzalez-Guerra, Actas Dermosifiliogr., 2014, 105, 833-839.

31 S. Harrison and R. Sinclair, J. Cosmet. Dermatol., 2003, 2, 180-185.

32 R. Dawber, Clin. Dermatol., 1996, 14, 105-112.

33 S. S. Adav, R. S. Subbaiaih, S. K. Kerk, A. Y. Lee, H. Y. Lai, K. W. Ng, S. K. Sze and A. Schmidtchen, Sci. Rep., 2018, 8, 1599.

34 S. Ito, Y. Nakanishi, R. K. Valenzuela, M. H. Brilliant, L. Kolbe and K. Wakamatsu, Pigm. Cell Melanoma Res., 2011, 24, 605-613.

35 S. Ito, K. Wakamatsu and T. Sarna, Photochem. Photobiol., 2018, 94, 409-420.

36 K. Wakamatsu, Y. Nakanishi, N. Miyazaki, L. Kolbe and S. Ito, Pigm. Cell Melanoma Res., 2012, 25, 434-445.

37 S. Ito, A. Pilat, W. Gerwat, C. M. Skumatz, M. Ito, A. Kiyono, A. Zadlo, Y. Nakanishi, L. Kolbe, J. M. Burke, T. Sarna and K. Wakamatsu, Pigm. Cell Melanoma Res., 2013, 26, 357366.

38 S. Li, D. Gao and Y. Jiang, Metabolites, 2019, 9, 36.

39 K. Foitzik, E. Hoting, T. Forster, P. Pertile and R. Paus, Exp. Dermatol., 2007, 16, 936-945.

40 G. Brotzu, A. M. Fadda, M. L. Manca, T. Manca, F. Marongiu, M. Campisi and F. Consolaro, Dermatol. Ther., 2019, 32, e12778.

41 J. Kluge, L. Rentzsch, D. Remane, F. T. Peters and D. K. Wissenbach, Drug Test. Anal., 2018, 10, 1536-1542.

42 A. H. Rashaid, P. B. Harrington and G. P. Jackson, Anal. Chem., 2015, 87, 7078-7084.

43 C. R. Robbins, in Chemical and Physical Behavior of Human Hair, 2012, ch. 2, pp. 105-176, DOI: 10.1007/978-3-64225611-0_2.

44 T. Iwabuchi, R. Ideta, R. Ehama, H. Yamanishi, M. Iino, Y. Nakazawa, T. Kobayashi, M. Ohyama and J. Kishimoto, J. Dermatol., 2016, 43, 567-570.

45 Y. Watanabe, T. Nagashima, N. Hanzawa, A. Ishino, Y. Nakazawa, M. Ogo, T. Iwabuchi and M. Tajima, Int. J. Cosmet. Sci., 2015, 37, 579-587.

46 M. Giorgio, G. I. Dellino, V. Gambino, N. Roda and P. G. Pelicci, Redox Biol., 2020, 29, 101398. 